\title{
A Susceptible Exposed Infected Recovered Susceptible (SEIRS) Model for the Transmission of Tuberculosis
}

\author{
Latifat M Erinle-Ibrahim ${ }^{1 *}$, Waheed $O$ Lawal ${ }^{1}$, Olukayode Adebimpe ${ }^{2}$ and Gbemisola $R$ \\ Sontan ${ }^{1}$ \\ ${ }^{I}$ Department of Mathematics, College of Science and Information Technology, Tai- \\ Solarin University of Education, P.M.B. 2118, Ijebu Ode, Nigeria. \\ ${ }^{2}$ Department of Physical Sciences, Land Mark University, Omu-Aran, Nigeria. \\ *Corresponding author's e-mail address: erinle-ibrahimlm@tasued.edu.ng \\ Co-authors'e-mail addresses: gbemmyrache60@gmail.com,waheedlawal207@yahoo.com, \\ adebimpe.olukayode@lmu.edu.ng
}

Received 15 Mar 2021, Revised 9 Jul 2021, Accepted 20 Jul 2021, Published Aug 2021

DOI: https://dx.doi.org/10.4314/tjs.v47i3.4

\begin{abstract}
In this paper, a deterministic mathematical model was proposed and analyzed to understand the dynamics of tuberculosis based on the SEIRS model. The disease-free equilibrium, the endemic equilibrium, and their stabilities were examined. The $\mathrm{R}_{0}$ (basic reproduction number) was derived using the Next Generation Matrix method and its sensitivity analysis showed that the birth rate and infectious rate were the most sensitive parameters of $R_{0}$. The behaviour of exposed individuals at the latent period with varied treatment rates were examined through numerical simulation. From the analysis carried out, the effect of variations of the treatments of latent TB shows that it affects the disease burden. This implies that testing and treatment of latent TB are important in preventing it from becoming infectious. The re-infection rate was examined to see the effect it had both on the recovered and susceptible populations. The study concludes by recommending the extension of the model to an age structured model with co-infection with another respiratory infectious disease like COVID-19.
\end{abstract}

Keywords: Epidemiology, Latent TB treatment, Basic Reproduction Number, sensitivity analysis, numerical simulation.

\section{Introduction}

Tuberculosis (TB) is an infectious disease that poses threats to the human population in the world. Tuberculosis is caused by bacteria generally referred to as Mycobacterium tuberculosis and almost every organ can be affected, but the involvement of the lungs accounts for more than $80 \%$ of TB cases. Tuberculosis affecting the lungs is called Pulmonary Tuberculosis (PTB), while those affecting the organs are called Extra Pulmonary Tuberculosis (ETB). The most important source of infection is an untreated Pulmonary
Tuberculosis (PTB). When such an infected one coughs, sneezes, talks, or spits, tiny droplets containing tuberculosis are released. Transmission is through inhaling those droplets (Federal Ministry of Health 2010).

According to the World Health Organization, the most vulnerable to Mycobacterium tuberculosis are children and infants because their immunity cannot yet withstand such infections. This infectious disease claimed the lives of 1.5 million people in 2018 (including 251,000 people with HIV). TB remains one of the foremost of the causes 
of death from just a single infectious disease. In 2018 , on a worldwide scale, 10 million fell ill due to tuberculosis. Of these 10 million, there were 5.7 million men, 3.2 million women, and 1.1 million children. Also, in the same year, the 30 high TB burden countries accounted for $87 \%$ of new TB cases. Eight countries accounted for two-thirds of the total, with India leading the count followed by China, Indonesia, the Philippines, Pakistan, Nigeria, Bangladesh, and South Africa (WHO 2019).

Diagnosis of latent TB infections (LTBI) and prompt treatments of infected cases are vital components of effective TB control. On the other hand, undetected TB infections and delay in the treatments of active TB cases can lead to more severe disease conditions in the infected persons which could result in wider disease spread in the community. Such delays also contribute to increased infectivity in the community, whereby the infected individuals unknowingly continue to serve as reservoirs for the pathogens (M. tuberculosis). Hence, this could lead to an increased risk of disease transmission in the community (Egonmwan and Okuonghae 2019). In the past two decades, the WHO has listed Nigeria as one of the countries with a high burden of $\mathrm{TB}$ and has stimulated targeted interventions and advocacy for funding and policies to improve TB control. It is estimated that 407,000 people in Nigeria have TB each year with a rate of 219 cases per 100,000 population with 106,533 total cases notified in 2018 (WHO 2019).

Tuberculosis also has serious social implications because those with TB and their families can face challenges in social relationships and adverse health and economic situations. People with TB and their relatives could experience prejudice and negative attitudes from people (Okuonghae and Ikhimwin 2016). In light of the alarming rates at which tuberculosis is being recorded every year, mathematical models to analyze the spread of TB have been widely developed. Epidemic models that could be stochastic or deterministic have allowed researchers to gain an understanding of many infectious diseases and they have also helped them to provide strategies for combating them. In this paper, compartment $S$ is such that everyone is susceptible, $E$ a latent compartment in which all the individuals are all infected but not yet infectious, and next is the $I$ compartment in which all in the compartment are infectious, which means they are showing some symptoms or all the symptoms of TB, and lastly the $R$ compartment where we have those that have recovered from TB due to treatments (Sontan 2020).

Many mathematical models have been developed and used to study the spread of TB in populations. For example, McCluskey and Driessche (2004) analyzed two tuberculosis models that included treatment of latent and infective individuals. Three parameters determined the existence and local stability of equilibria. Also, Egonmwan and Okuonghae (2019) modelled the spread of TB and the results of the numerical simulations of the model indicated that the treatment rates for latent and active TB cases significantly determine the impacts of the fraction of new latent TB cases diagnosed.

The structure of the paper is such that: we formulate a simple Ordinary Differential Equation (ODE) model, find the Disease-Free equilibrium, and Endemic equilibrium, then we derive the $R_{0}$, and the sensitivity analysis.

The result of the basic reproduction number $\boldsymbol{R}_{\boldsymbol{0}}$ was estimated using the next generation matrix method. The sensitivity analysis results showed that $\mathrm{B}$ (birth rate) and $\beta$ (transmission rate) have the most direct effects on $\boldsymbol{R}_{\boldsymbol{\theta}}$ which can be used to contain the spread of TB. The work also confirms that once someone is treated for $\mathrm{TB}$, it does not mean the individual is immunized for life, but the person can be reinfected if the immunity is lost or the person is re-exposed. In conclusion, latent treatment of TB helps in preventing it from becoming infectious, hence testing for latent $\mathrm{TB}$ is important for those with compromised immunity. 


\section{Materials and Methods}

The mathematical model formulated by Momoh et al. (2013) was modified to incorporate the rate at which the recovered return to susceptible because $\mathrm{TB}$ does not confer lifelong immunity. Also, the infectious rate $\beta$ (the rate at which the susceptible become infected) is a function of the number of infectious hosts in the population at time $t$, and thus it is a non-linear term - $\beta S I$. Other transitions were modeled as linear terms with a constant coefficient. Therefore, the TB dynamics were described by a system of ODE which was solved to obtain the disease-free equilibrium (DFE) state and endemic state. The stability analysis of the DFE was carried out using the Jacobian matrix and the endemic stability using the $R_{0}$.

\section{Model equations}

As shown in the compartmental model in Figure 1, and with the described variables and parameters in Tables 1 and 2, respectively, we have the following system of differential equations:

$$
\begin{aligned}
& \frac{d S}{d t}=B-S(\beta I+\mu)+\xi R \\
& \frac{d E}{d t}=\beta S I-E(\delta+\mu+\alpha) \\
& \frac{d I}{d t}=\alpha E-I(\gamma+\mu) \\
& \frac{d R}{d t}=\gamma I+\delta E-R(\xi+\mu) \\
& N(t)=S(t)+E(t)+I(t)+R(t)
\end{aligned}
$$

\begin{tabular}{|c|c|c|}
\hline Variable & Description & \\
\hline $\mathrm{S}(\mathrm{t})$ & \multicolumn{2}{|c|}{ The number of susceptible individuals at time $\mathrm{t}$} \\
\hline $\mathrm{E}(\mathrm{t})$ & \multicolumn{2}{|c|}{ The number of exposed individuals at time $t$} \\
\hline $\mathrm{I}(\mathrm{t})$ & \multicolumn{2}{|c|}{ The number of infected individuals at time $t$} \\
\hline $\mathrm{R}(\mathrm{t})$ & \multicolumn{2}{|c|}{ The number of recovered individuals at time $t$} \\
\hline $\mathrm{N}(\mathrm{t})$ & The total number of population & \\
\hline Parameter & Description & \\
\hline $\mathrm{B}$ & Birth rate & \\
\hline$\mu$ & Natural death rate & \\
\hline$\beta$ & Infectious rate & \\
\hline$\alpha$ & \multicolumn{2}{|c|}{ Disease progression rate from latent $\mathrm{TB}$ to active TB } \\
\hline $\begin{array}{l}\delta \\
\gamma\end{array}$ & \multicolumn{2}{|c|}{$\begin{array}{l}\text { Treatment rate for individuals with latent TB } \\
\text { Treatment rate of actively infected TB }\end{array}$} \\
\hline$\xi$ & Rate of re-infection & \\
\hline \multicolumn{3}{|c|}{ Table 2: Parameter values } \\
\hline $\mathbf{B}$ & 0.03768 & Estimated \\
\hline$\mu$ & 0.02041 & Estimated \\
\hline$\beta$ & 6.55000 & Egonmwan and Okuonghae (2019) \\
\hline$\alpha$ & 0.00050 & Egonmwan and Okuonghae (2019) \\
\hline$\delta$ & 1.50 & Egonmwan and Okuonghae (2019) \\
\hline$\gamma$ & 2.50 & Assumed \\
\hline$\xi$ & 1.20 & Assumed \\
\hline
\end{tabular}




\section{The mathematical model and diagram}

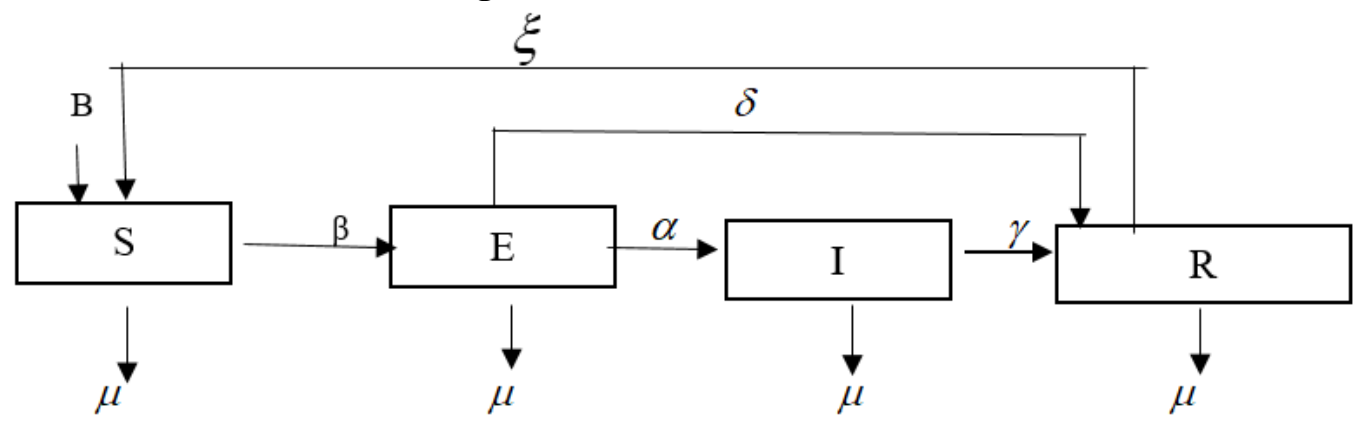

Figure 1: The transmission dynamics of the tuberculosis model is described by the compartment model.

\section{Results}

Disease-free equilibrium state

We obtain the disease-free equilibrium state by setting Equations (2) -(4) to be equal to zero.

$$
\begin{aligned}
& \frac{d E}{d t}=\frac{d I}{d t}=\frac{d R}{d t}=0 \\
& \beta S I-\delta E-\mu E-\alpha E=0 \\
& \alpha E-\gamma I-\mu I=0 \\
& \gamma I-\delta E-\xi R-\mu R=0 \\
& S=N=\frac{B}{\mu}
\end{aligned}
$$

Therefore, we have the disease-free equilibrium

$$
E_{0}=(S, E, I, R)=\left(\frac{B}{\mu}, 0,0,0\right)
$$

\section{Basic reproduction number $\boldsymbol{R}_{\boldsymbol{0}}$}

The basic reproduction number $R_{0}$ was obtained with the method of the next generation matrix. We created a sub-model that only considers the 'disease' compartments (Diekmann et al. 2010). For the SEIRS model, a subset of the system includes both the exposed and infectious individuals. Therefore, the sub-model will only contain the $E$ and $I$ equations.

$$
\frac{d \vec{x}}{d t}=F(\vec{x})-V(\vec{x})
$$

$\vec{x}$ is a vector of the $j$ disease compartments in the SEIRS model $j=2$, because the disease compartments are two, that is $E$ and $I$.

$$
\frac{d \vec{x}}{d t}=\left(\begin{array}{c}
\beta S I \\
0
\end{array}\right)-\left(V^{-}(\vec{x})-V^{+}(\vec{x})\right)=\left(\begin{array}{c}
\beta S I \\
0
\end{array}\right)-\left(\left[\begin{array}{c}
(\delta+\mu+\alpha) E \\
(\gamma+\mu) I
\end{array}\right]-\left[\begin{array}{c}
0 \\
\alpha E
\end{array}\right]\right)
$$

Next, we linearize around the DFE, which involves the Jacobian, i.e., the matrix of the partial derivative evaluated at the DFE.

The disease-free equilibrium $\left(E_{0}\right)$ is given by:

$$
(S, E, I, R)=\left(\frac{\beta}{\mu}, 0,0,0\right)
$$

$$
\begin{aligned}
& J(S, E, I, R)=\left[\begin{array}{ll}
\partial F 1 / \partial E & \partial F 1 / \partial I \\
\partial F 2 / \partial E & \partial F 2 / \partial I
\end{array}\right]_{\left(\frac{\beta}{\mu}, 0,0,0\right)} \\
& =\left[\begin{array}{cc}
-(\delta+\mu+\alpha) & \beta S \\
\alpha & -(\alpha+\mu)
\end{array}\right]_{\left(\frac{\beta}{\mu}, 0,0,0\right)}
\end{aligned}
$$




$$
\begin{aligned}
& =\left[\begin{array}{cc}
-(\delta+\mu+\alpha) & \frac{\beta B}{\mu} \\
\alpha & -(\alpha+\mu)
\end{array}\right] \\
& {\left[\begin{array}{c}
\frac{d E}{d t} \\
\frac{d I}{d t}
\end{array}\right]=\left(\left[\begin{array}{cc}
0 & \frac{\beta B}{\mu} \\
0 & 0
\end{array}\right]-\left[\begin{array}{cc}
(\delta+\mu+\alpha) & 0 \\
-\alpha & \gamma+\mu
\end{array}\right]\right)\left(\begin{array}{l}
E \\
I
\end{array}\right)} \\
& F=\left[\begin{array}{cc}
0 & \frac{\beta B}{\mu} \\
0 & 0
\end{array}\right) \\
& V=\left[\begin{array}{cc}
(\delta+\mu+\alpha) & 0 \\
-\alpha & \gamma+\mu
\end{array}\right]
\end{aligned}
$$

Where $F V^{-1}$ is known as the next generation matrix

$$
\begin{aligned}
& F V^{-1}=\left(\begin{array}{cc}
\frac{\beta B \alpha}{\mu(\delta+\mu+\alpha)} & \frac{\beta B}{\mu(\gamma+\mu)} \\
0 & 0
\end{array}\right) \\
& R_{0}=\rho\left(F V^{-1}\right)
\end{aligned}
$$

$R_{0}$ is the spectra radius of the next generation matrix. $\rho$ is the eigenvalues with the largest magnitude. Next, we find the eigenvalues of Equation (15);

$$
\begin{aligned}
& |J-\lambda I|=0 \\
& \left|\begin{array}{cc}
\frac{B \beta \alpha}{\mu(\delta+\mu+\alpha)}-\lambda & \frac{\beta B}{\mu(\gamma+\mu)} \\
0 & 0-\lambda
\end{array}\right|=0 \\
& \lambda_{1}=0 \\
& \lambda_{2}=\frac{B \beta \alpha}{\mu(\delta+\mu+\alpha)(\gamma+\mu)}
\end{aligned}
$$

The Eigenvalue with the largest magnitude is

$$
\frac{B \beta \alpha}{\mu(\delta+\mu+\alpha)(\gamma+\mu)}
$$

Hence, $R_{0}=\frac{B \beta \alpha}{\mu(\delta+\mu+\alpha)(\gamma+\mu)}$

\section{The endemic equilibrium state}

The endemic equilibrium state is the condition where the disease persists in the population. For the disease to persist in the population, the susceptible class, the exposed class, the infectious, and the recovered must not be zero at equilibrium state (Ugwa et al. 2013). In other words, if $\left(S^{*}, E^{*}, I^{*}, R^{*}\right)$ is the endemic equilibrium state, then $\left(S^{*}, E^{*}, I^{*}, R^{*}\right) \neq(0,0,0,0)$. To obtain the endemic equilibrium, we solve Equations (1) (4) simultaneously.

$$
S^{*}=\frac{(\gamma+\mu)(\delta+\mu+\alpha)}{\beta \alpha}
$$

But writing it in terms of $R_{0}$ in Equation (16)

$$
\begin{gathered}
\boldsymbol{S}^{*}=\frac{\boldsymbol{B}}{\boldsymbol{\mu} \boldsymbol{R}_{\mathrm{O}}} \\
E^{*}=\frac{(\gamma+\mu)\left[(\mu+\xi) B \mu\left(R_{0}-1\right)\right]}{\alpha\left((\xi+\mu) B \beta-R_{0} \xi \mu\left(\gamma+\delta\left(\frac{\gamma+\mu}{\alpha}\right)\right)\right)} \\
I^{*}=\frac{(\mu+\xi) B \mu\left(R_{0}-1\right)}{\left((\xi+\mu) B \beta-R_{0} \xi \mu\left(\gamma+\delta\left(\frac{\gamma+\mu}{\alpha}\right)\right)\right)} \\
R^{*}=\frac{B \mu\left(R_{0}-1\right)\left(\gamma+\delta \frac{(\gamma+\mu)}{\alpha}\right)}{(\xi+\mu) B \beta-R_{0} \xi \mu\left(\gamma+\frac{\delta(\gamma+\mu)}{\alpha}\right)}
\end{gathered}
$$

Therefore, the endemic equilibrium state is $\left(S^{*}, E^{*}, I^{*}, R^{*}\right)$.

\section{Local stability of disease- free equilibrium}

Theorem 1: If $R_{0}<1$, the disease-free equilibrium of systems (1-4) is locally asymptotically stable. If $R_{0}>1$, the diseasefree equilibrium is unstable.

Proof: The disease- free equilibrium state is given as: 


$$
E_{0}=\left(\frac{\beta}{\mu}, 0,0,0\right)
$$

The resulting Jacobian matrix of the systems (1-4) is:

$$
J\left(E_{0}\right)=\left(\begin{array}{cccc}
-(\beta I+\mu) & 0 & -\beta S & \xi \\
\beta I & -(\delta+\mu+\alpha) & \beta S & 0 \\
0 & \alpha & -(\gamma+\mu) & 0 \\
0 & \delta & \gamma & -(\xi+\mu)
\end{array}\right)
$$

Substituting the values of (21) into (22)

$$
\begin{aligned}
& S^{*}=\frac{\beta}{\mu}, E^{*}=0, I^{*}=0, R^{*}=0 \\
& J\left(E_{0}\right)=\left(\begin{array}{cccc}
-\mu & 0 & \frac{-B \beta}{\mu} & \xi \\
0 & -(\delta+\mu+\alpha) & \frac{B \beta}{\mu} & 0 \\
0 & \alpha & -(\gamma+\mu) & 0 \\
0 & \delta & \gamma & -(\xi+\mu)
\end{array}\right)
\end{aligned}
$$

To evaluate this, the Eigenvalues are introduced such that $|J-\lambda I|=0$

$$
-\mu-\lambda\left|\begin{array}{ccc}
-(\delta+\mu+\alpha)-\lambda & \frac{B \beta}{\mu} & 0 \\
\alpha & -(\gamma+\mu)-\lambda & 0 \\
\delta & \gamma & -(\xi+\mu)-\lambda
\end{array}\right|=0
$$

The characteristics equation is given as:

$-\mu-\lambda\left[(-(\delta+\mu+\alpha)-\lambda(-\gamma+\mu)-\gamma)(-(\xi-\mu)-\lambda)-\frac{B \beta}{\mu}(\alpha(\xi-\mu-\lambda))\right]$

The eigenvalues are:

$\lambda_{1}=-\mu$

$\lambda_{2}=-(\xi+\mu)$

The resulting quadratic equation is:

$\lambda^{2}+(2 \mu+\delta+\alpha+\gamma) \lambda+\mu^{2}+\delta \gamma+\delta \mu+\mu \gamma+\alpha \gamma+\alpha \mu-\alpha \beta \frac{B}{\mu}=0$

For negative roots, Descartes' rule of signs,

$\mu^{2}+\delta \gamma+\delta \mu+\mu \gamma+\alpha \gamma+\alpha \mu-\alpha \beta \frac{B}{\mu}=0$ 
$1>\frac{B \beta \alpha}{\mu(\delta+\mu+\alpha)(\gamma+\mu)}$

But from (16) $R_{0}=\frac{B \beta \alpha}{\mu(\delta+\mu+\alpha)(\gamma+\mu)}$

If $\lambda_{1}<0, \lambda_{2}<0$, and $R_{0}<1$, the disease-free equilibrium is asymptotically stable.

\section{Local stability of endemic equilibrium}

Theorem 2: If $R_{0}>1$, the endemic equilibrium $E^{*}$ of systems (1-4) is locally asymptotically stable.

Proof: The Jacobian matrix of systems (1-4) at the endemic equilibrium $E^{*}$ is:

$$
J\left(E^{*}\right)=\left|\begin{array}{cccc}
-\left(\beta I_{*}+\mu+\lambda\right) & 0 & -\beta S_{*} & \xi \\
\beta I_{*} & -(\delta+\mu+\alpha+\lambda) & \beta S_{*} & 0 \\
0 & \alpha & -(\gamma+\mu+\lambda) & 0 \\
0 & \delta & \gamma & -(\xi+\mu+\lambda)
\end{array}\right|=0
$$

The eigen value matrix $J\left(E^{*}\right)$ is $\lambda_{1}=-(\xi+\mu)$ and the resulting polynomial is:

$\lambda^{3}+\left(3 \mu+\gamma+\alpha+\delta+\beta I_{*}\right) \lambda^{2}+\left(2 \alpha \mu+\alpha \gamma+3 \mu^{2}+2 \mu \gamma+2 \delta \mu+\delta \mu+\beta I_{*} \alpha+\beta I_{*} \gamma+2 \beta I_{*} \mu+\beta I_{*} \delta-\beta S_{*} \mu \alpha\right) \lambda$

$$
\beta I_{*} \delta \gamma+\beta I_{*} \delta \mu+\beta I_{*} \mu \gamma+\beta I_{*} \mu^{2}+\beta I_{*} \alpha \gamma+\beta I_{*} \alpha \mu+\mu \delta \gamma+\mu^{2} \delta+\mu^{2} \gamma+\mu^{3}+\mu \alpha \gamma+\mu^{3} \alpha-\beta I_{*} \mu \alpha=0
$$

The above polynomial could be written in this form:

$$
\begin{aligned}
& a_{3} \lambda^{3}+a_{2} \lambda^{2}+a_{1} \lambda+a_{o}=0 \text { where, } \\
& a_{3}=1, \quad a_{2}=\left(3 \mu+\gamma+\alpha+\delta+\beta I_{*}\right), \\
& \quad a_{1}=\left(2 \alpha \mu+\alpha \gamma+3 \mu^{2}+2 \mu \gamma+2 \delta \mu+\delta \mu+\beta I_{*} \alpha+\beta I_{*} \gamma+2 \beta I_{*} \mu+\beta I_{*} \delta-\beta S_{*} \mu \alpha\right), \\
& a_{0}=\beta I_{*} \delta \gamma+\beta I_{*} \delta \mu+\beta I_{*} \mu \gamma+\beta I_{*} \mu^{2}+\beta I_{*} \alpha \gamma+\beta I_{*} \alpha \mu+\mu \delta \gamma+\mu^{2} \delta+\mu^{2} \gamma+\mu^{3}+\mu \alpha \gamma+\mu^{3} \alpha-\beta I_{*} \mu \alpha
\end{aligned}
$$

By Routh- Hurwitz stability criterion for third order polynomials $a_{2} a_{1}>a_{3} a_{0}$. Hence the local stability of endemic equilibrium is locally asymptotically stable.

\section{Sensitivity analysis}

The sensitivity analysis helps to show how important every parameter is to disease transmission. It is regularly used to decide the robustness of model expectations to parameter values since there may be errors in data collection and assumed values (Berhe et al. 2019). The normalized forward sensitivity index of a variable regarding a parameter is the proportion of the relative change in the variable to the relative change in the parameter. The normalized forward sensitivity index $R_{0}$ that depends differentially on a parameter $\rho$ is defined by:

$\varphi_{\rho}^{R_{0}}=\frac{\partial R_{0}}{\partial \rho} \frac{\rho}{R_{0}}$

From Equation (16)

$$
\begin{aligned}
& R_{0}=\frac{B \beta \alpha}{\mu(\delta+\mu+\alpha)(\gamma+\mu)} \\
& \varphi_{B}^{R_{0}}=\frac{\partial R_{0}}{\partial B} \frac{B}{R_{0}}
\end{aligned}
$$




$$
\begin{aligned}
\varphi_{B}^{R_{0}} & =+1 \\
\varphi_{\beta}^{R_{0}} & =\frac{\partial R_{0}}{\partial \beta} \frac{B}{R_{0}} \\
\varphi_{\beta}^{R_{0}} & =+1 \\
\varphi_{\alpha}^{R_{0}} & =\frac{\partial R_{0}}{\partial \alpha} \frac{\alpha}{R_{0}} \\
\varphi_{\alpha}^{R_{0}} & =\frac{\delta+\mu}{\delta+\mu+\alpha} \\
\varphi_{\mu}^{R_{0}} & =\frac{\partial R_{0}}{\partial \mu} \frac{\mu}{R_{0}} \\
\varphi_{\mu}^{R_{0}}= & \frac{-\left(3 \mu{ }^{2}+2 \mu(\alpha+\gamma+\delta)+\gamma(\alpha+\delta)\right)}{(\delta+\mu+\alpha)(\gamma+\mu)} \\
\varphi_{\delta}^{R_{0}} & =\frac{\partial R_{0}}{\partial \delta} \frac{\delta}{R_{0}} \\
\varphi_{\delta}^{R_{0}} & =\frac{-\delta}{(\delta+\mu+\alpha)} \\
\varphi_{\gamma}^{R_{0}} & =\frac{-\gamma}{\gamma+\mu} \\
\varphi_{\gamma} & =\frac{\partial R_{0}}{\partial \gamma} \frac{\gamma}{R_{0}}
\end{aligned}
$$

Numerical solution of sensitivity analysis of the basic reproduction number $\boldsymbol{R}_{\boldsymbol{\theta}}$

Table 3: The sensitivity of basic reproduction number $\boldsymbol{R}_{\boldsymbol{0}}$

\begin{tabular}{lll}
\hline Parameters & Signs & $\begin{array}{l}\text { Sensitivity } \\
\text { Values }\end{array}$ \\
\hline $\mathrm{B}$ & + & 1 \\
$\beta$ & + & 1 \\
$\alpha$ & + & 0.9996712494 \\
$\mu$ & - & -1.021517486 \\
$\delta$ & - & -0.9862516520 \\
$\gamma$ & - & -0.9919021112 \\
\hline
\end{tabular}

From table 3 , the sensitivity indices of $\varphi_{B}^{R_{0}}, \varphi_{\beta}^{R_{0}}, \varphi_{\alpha}^{R_{0}}$ are all positive, while the remaining parameters are negative. Since all indices are functions of other parameters, the sensitivity indices will change when other parameter values change. The value of $\varphi_{B}^{R_{0}}$ $=+1$ means that increasing (or decreasing) $R_{0}$ by $10 \%$ increases (or decreases) $R_{0}$ by $10 \%$. The same is true for $\varphi_{\beta}^{R_{0}}$. Both have exactly the same effect on the basic reproduction number. However, the rest parameters too have approximately the same effect on $\mathrm{R}_{0}$ which implies that they all have to be carefully estimated.

\section{Numerical simulation}

The system of equations (1-4) cannot be solved analytically due to non-linearity, so the set of equations were solved using a numerical method. The finite difference method was used. The initial conditions $\mathrm{S}(0)=990, \mathrm{E}(0)=8$, I $(0)=2, \mathrm{R}(0)=0$ at $\mathrm{t}=0, \mathrm{~h}=0.05$

$f^{\prime}(x) \approx \frac{f(x+h)-f(x)}{h}$

Discussion

Figure 2 shows the dynamics of the compartments population over time. We observed that the susceptible population was decreasing with time due to their moving to other compartments. The exposed population showed increasing and later decreasing due to a latent stage treatment. The infected population showed decreasing due to good treatment rate and hence people recovering. The rate of recovery over time is high. This is as a result of those with latent TB getting tested and treated and those with infectious $\mathrm{TB}$ receiving adequate treatments. All these happen simultaneously in a population when an infectious disease is in a population over time. Figure 3 shows the impacts of the treatments of latent $\mathrm{TB}$ on the exposed population. The treatment rates were steadily increased from $\delta$ $=1.5$ to $\delta=2$ to $\delta=2.5$ and $\delta=3$. The effect on the exposed population was examined and the exposed population reduced from approximately 350 people to 227 people, then to 200 people and to 159 people at the end of 
the simulated time. This shows that early diagnosis and treatment of latent TB helps in reducing the exposed population over time. Figure 4 shows the effects of TB re-infections on the recovered and susceptible populations. The re-infection rates were reduced from $\xi=$ 1.5 to $\xi=0.9$ to $\xi=0.6$ and $\xi=0.3$. The implication is that the recovered population increased from about 450 people to 503 people and further to 565 , and then to 638 people, respectively at the end of the simulated time. Also, the susceptible population decreased from 167 people to 137 people and further to 100 , and finally to 55 people at the end of the simulated time. As the re-infection rate- $\xi$ decreases, the recovered population increases, and the susceptible decreases. This implies that if they prevent re-infection by avoiding being exposed again and maintaining good immunity, they remain recovered.

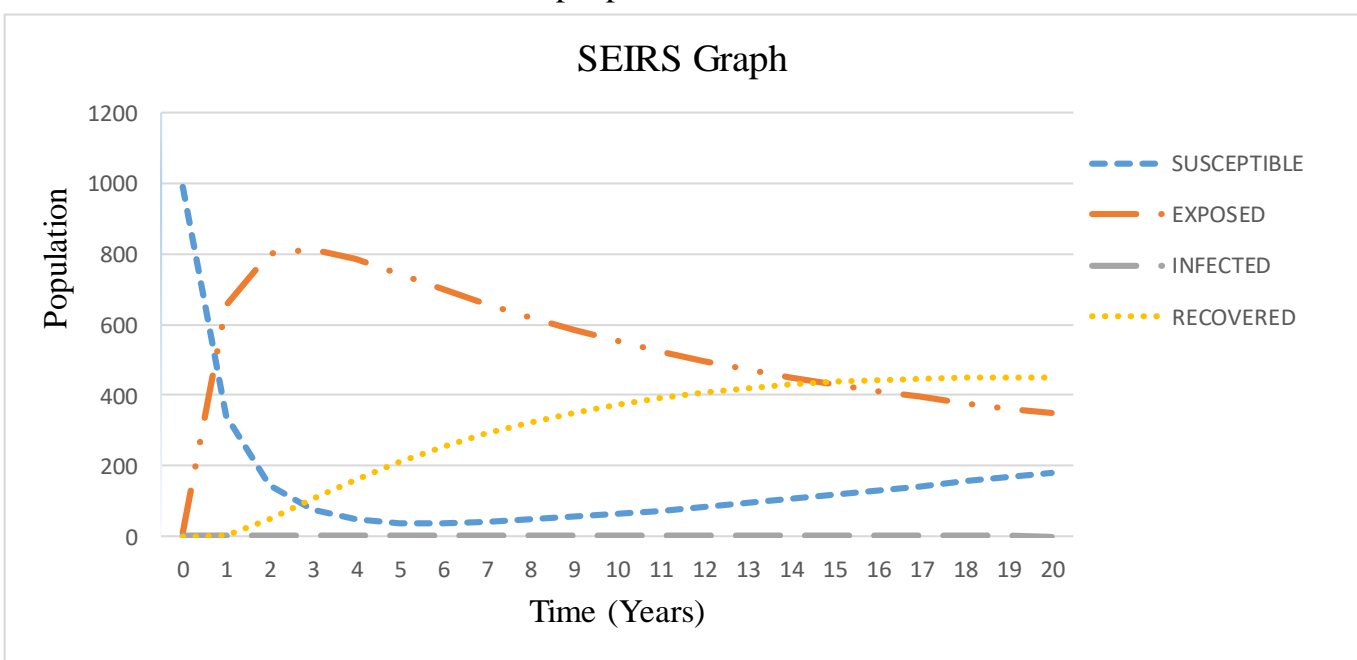

Figure 2: The SEIRS graph with the values of parameters on Table 2.

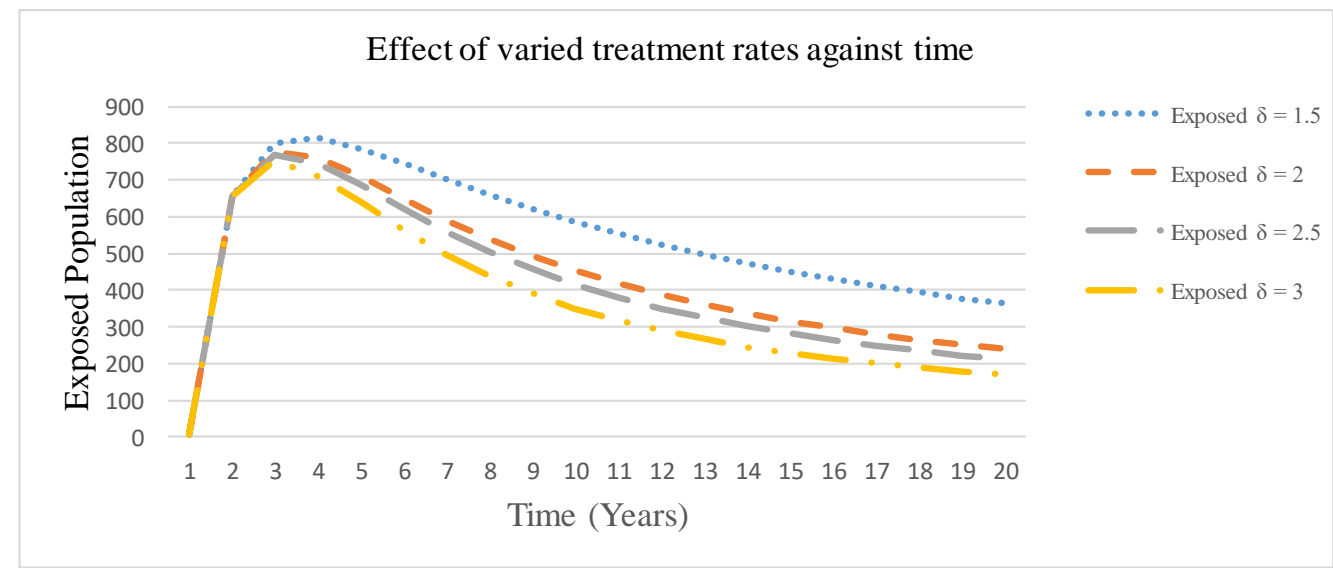

Figure 3: The graph of exposed population with treatment rates $\delta=1.5, \delta=2, \delta=2.5, \delta=3$ and other parameters fixed. 
Erinle-Ibrahim et al. - A Susceptible Exposed Infected Recovered Susceptible (SEIRS) ...

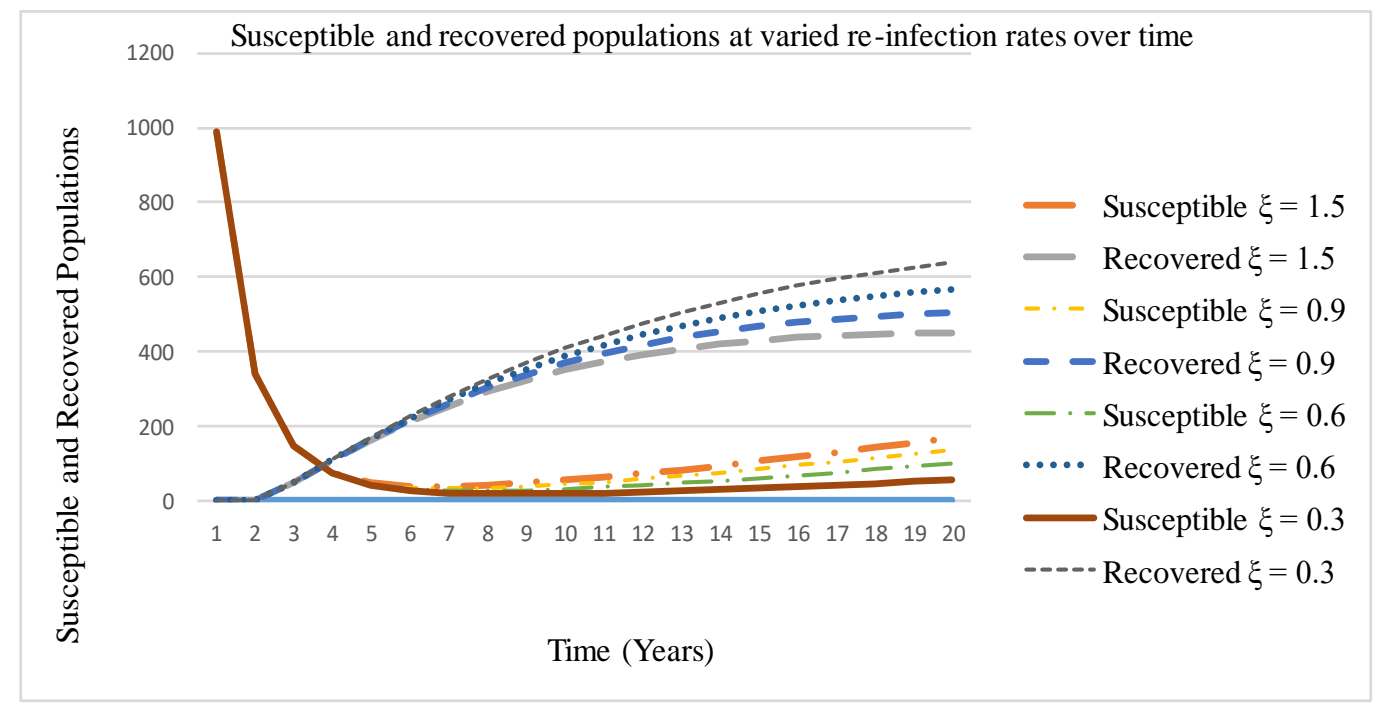

Figure 4: The graph of susceptible and recovered populations over time with rates of reinfection $\xi=1.5, \xi=0.9, \xi=0.6, \xi=0.3$ and other parameters fixed.

\section{Conclusion and Recommendations}

From the analysis carried out, the effect of variation of the treatments of latent $\mathrm{TB}$ showed that it affects the disease burden. This implies that testing and treatment of latent $\mathrm{TB}$ are important in preventing it from becoming infectious. Also, the re-infection rate was examined to see the effect it had on the susceptible and recovered population. It was noticed that when the re-infection rate was reduced, many people remained recovered. This implies the need for those who have been treated to avoid being re-infected.

It is hereby recommended that

- Latent TB testing should be made compulsory for those who have compromised immunity diseases like HIV, Cancer, etc.

- The susceptible class must be vaccinated at birth to prevent exposure.

- Those with TB should remain isolated until they start the treatments of TB.

- There should be ongoing public awareness and sensitization programmes on the effects of TB.

- More realistic models that include more compartments and co-infections with respiratory diseases like COVID 19 in age structured models should be examined.

\section{References}

Diekmann O, Heesterbeek JAP and Roberts MG 2010 The construction of nextgeneration matrices for compartmental epidemic models. J. Royal Soc. Interf. 7(47): 873-885.

Egonmwan AO and Okuongbae D 2019 Analysis of mathematical model for tuberculosis with diagnosis. J. Appl. Math. Comput. 59(1): 129-162.

Federal Ministry of Health 2010 Department of Public Health National Tuberculosis and Leprosy Control Programme, Worker's Manual.

Berhe HW, Makinde OD and Theuri DM 2019 Parameter estimation and sensitivity analysis of dysentery diarrhea epidemic model. J. Appl. Math. 2019: 1-13.

McCluskey CC and Driessche PVD 2004 Global analysis of two tuberculosis models. J. Dyn. Differ. Equations 16: 139-166.

Momoh AA, Ibrahim MO, Uwanta IJ and Manga SB 2013 Mathematical model for 
the control of measles epidemiology. Int. J. Pure Appl. Math. 87(5): 707-717.

Okuonghae D and Ikhimwin BO 2016

Dynamics of a mathematical model for tuberculosis with variability in susceptibility and disease progressions due to difference in awareness level. Front. Microbiol. 6(1530): 1-23.

Sontan GR 2020 A susceptible exposed infected recovered susceptible (SEIRS) model with dynamics for the transmission of tuberculosis. Master's dissertation, Tai-
Solarin University of Education Ijagun, Ijebu-Ode.

Ugwa KA, Agwu IA and Agbanyim AN 2013

Mathematical analysis of endemic equilibrium of the transmission dynamics of tuberculosis. Int. J. Sci. Technol. Res. 2(12): 263-269.

WHO (World Health Organization) 2019 Tuberculosis https://www.who.int/newsroom/facts/detail/tuberculosis 